\title{
Transverse malocclusion, posterior crossbite and severe discrepancy*
}

\author{
Roberto Carlos Bodart Brandão**
}

\begin{abstract}
This article reports the orthodontic treatment of a 14 years and 2 months old female patient, presenting both anterior and posterior unilateral left crossbite, related to a transverse atrophy of the maxilla and a severe negative tooth-arch discrepancy in the upper arch. A maxillary expansion with a modified Haas appliance was the first therapeutic attempt. Then, fixed appliances were used in both arches, and the second left premolar was extracted. The space for the upper left lateral incisor was achieved with compressed springs and tooth movement was accomplished with double archwires. The final result showed a good intercuspation, considering that the left molar relation remained as a Class II, which demanded special occlusal adjustments. This case was presented to the Brazilian Board of Orthodontics and Facial Orthopedics, representing category 5, as a partial requirement for the $\mathrm{BBO}$ certificate.
\end{abstract}

Keywords: Crossbite. Ectopic. Corrective orthodontics.

\section{HISTORY AND ETIOLOGY}

An orthodontic evaluation was first performed when the patient was 13 years old, but treatment only began when she was 14 years and 2 months old according to her parents' decision. Tooth crowding on the upper arch, with aesthetic consequences to her smile, was the chief complaint. She presented favorable con- ditions for treatment, free of caries and restorations, reasonably good oral hygiene, even on the upper left lateral incisor, rotated towards the palate. Good general health conditions were observed. She had good general health and it was also reported that her mother was submitted to an orthodontic treatment that required extraction of four premolars.
How to cite this article: Brandão RCB. Transverse malocclusion, posterior crossbite and severe discrepancy. Dental Press J Orthod. 2012 JanFeb;17(1):148-58.
" The author reports no commercial, proprietary, or financial interest in the products or companies described in this article.

* Clinical case report, category 5, approved by the Brazilian Board Orthodontics and Facial Orthopedics.

** PhD in Orthodontics, UNESP/Araraquara. MSc in Orthodontics, UFRJ. Professor of Orthodontics at UFES. Professor at FAESA Orthodontic

Specialization Program. Diplomate of the Brazilian Board of Orthodontics and Dentofacial Orthopedics (BBO). 


\section{DIAGNOSIS}

The greatest problems were seen in dental analysis. Molars and premolars were edge to edge bilaterally. There was a severe upper crowding, that resulted in the ectopic eruption of tooth \#22, palatally rotated and in crossbite. Upper canines were in infraocclusion, with the left side presenting semi-impaction and buccal rotation. Other than that, a moderate buccal inclination of the lower incisors was also observed (Fig 1).

The upper arch asymmetry deserved special attention. The midline was deviated $3 \mathrm{~mm}$ to the left, with a severe distal inclination of tooth \#21, further compromising her smile aesthetics (Fig 1). Using the Schmutz's plate, it was clear that the whole upper left side was $3 \mathrm{~mm}$ mesial in relation to the right side. An upper lip frenum fibrous attachment was also detected.

Patient presented a good lower arch regarding shape and symmetry, with the lower right first molar revealing an increased buccal root torque, that indicated some sort of constriction on the lower right side (Fig 2). The patient also presented a severe overbite, with the incisal edges of the
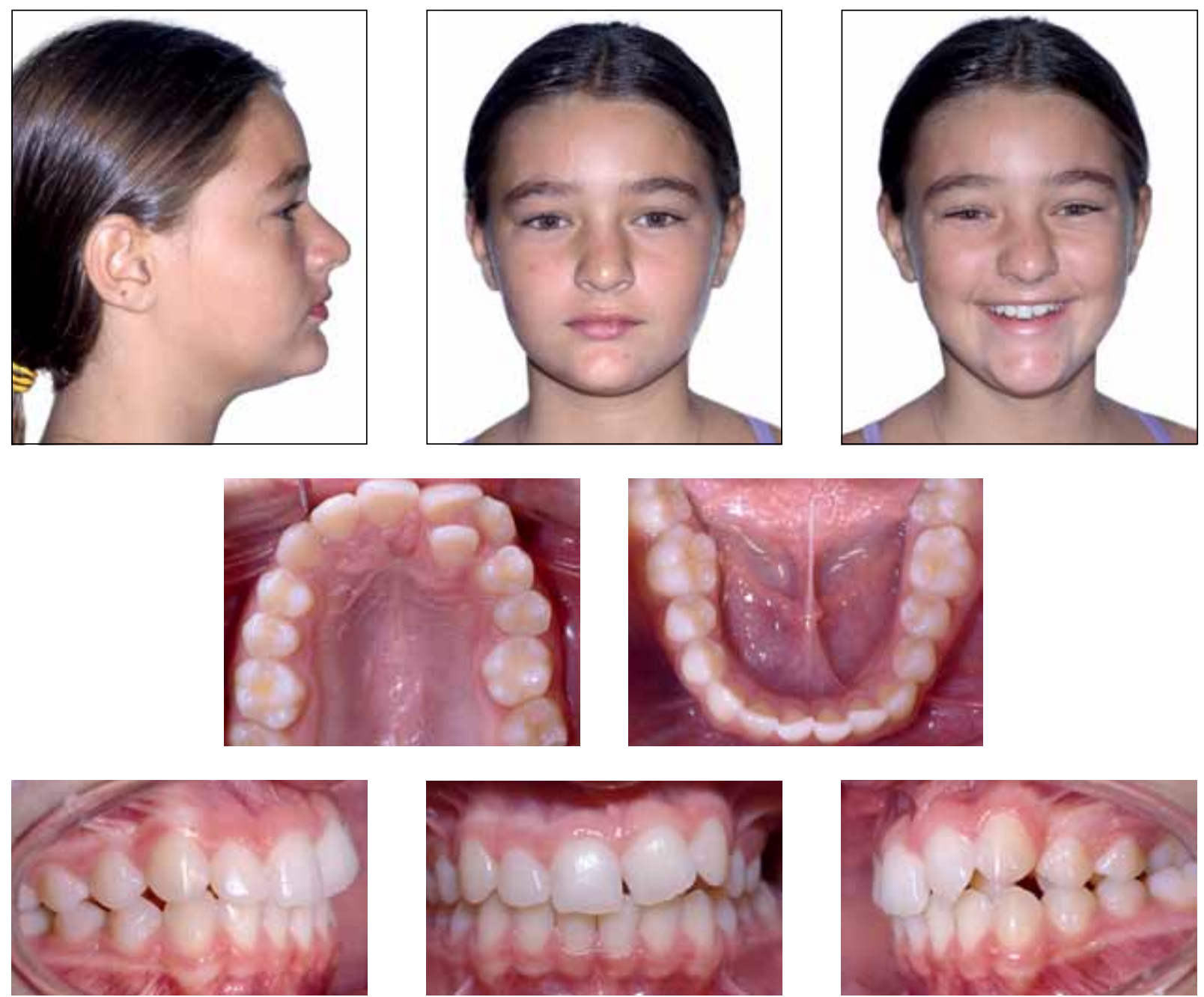

FIGURE 1 - Initial facial and intraoral photographs. 
lower incisors touching the cervical third of the lingual surface of the upper incisors.

Clinical assessment of the smile revealed a wide buccal corridor, confirmed with the occlusal dental casts analysis, with a moderate transverse constriction of the maxillary arch. This transverse problem was an important cause of the posterior left crossbite (Figs 1, 2 and 3).

Skeletal analysis revealed good maxillomandibular relationship at the anteroposterior aspect $\left(\mathrm{ANB}=2^{\circ}\right)$. For the vertical aspect, the patient was considered brachyfacial defined by the angles $\mathrm{SN}-\mathrm{GoGn}=24^{\circ}$ and $\mathrm{FMA}=18^{\circ}$ (Fig 4).

The concave facial profile represented a challenge both due to a high nasal prominence and a significant soft tissue thickness at the chin level. ${ }^{9}$ The chief complaint was eminently aesthetic, described as a "canted smile", strictly linked to the space collapse at the anterior part of the upper left side. In this case, the smile line inclination was mainly due to: 1) the inclination of the upper left central incisor; and 2) to the infraocclusion of the upper left canine, semi-impacted, rather than to the midline deviation.

\section{TREATMENT GOALS}

The treatment had as a primary goal the establishment of a better transverse dimension of the maxillary arch by means of skeletal expansion and, consequently to obtain the correction of the posterior crossbite. ${ }^{4}$ For the aesthetic improvement it would be necessary to open space for the alignment of the upper left lateral incisor, together with the uprighting of all upper anterior teeth in relation to the maxillary skeletal base. The smile line inclination would be finally reached by the extrusion of the upper left canine, provided the dominance of the upper central incisors was still preserved. ${ }^{6}$ Gaining canine disocclusion would be the main occlusal objective, as to reach effective occlusal determinants and anterior occlusal guidance, with immediate
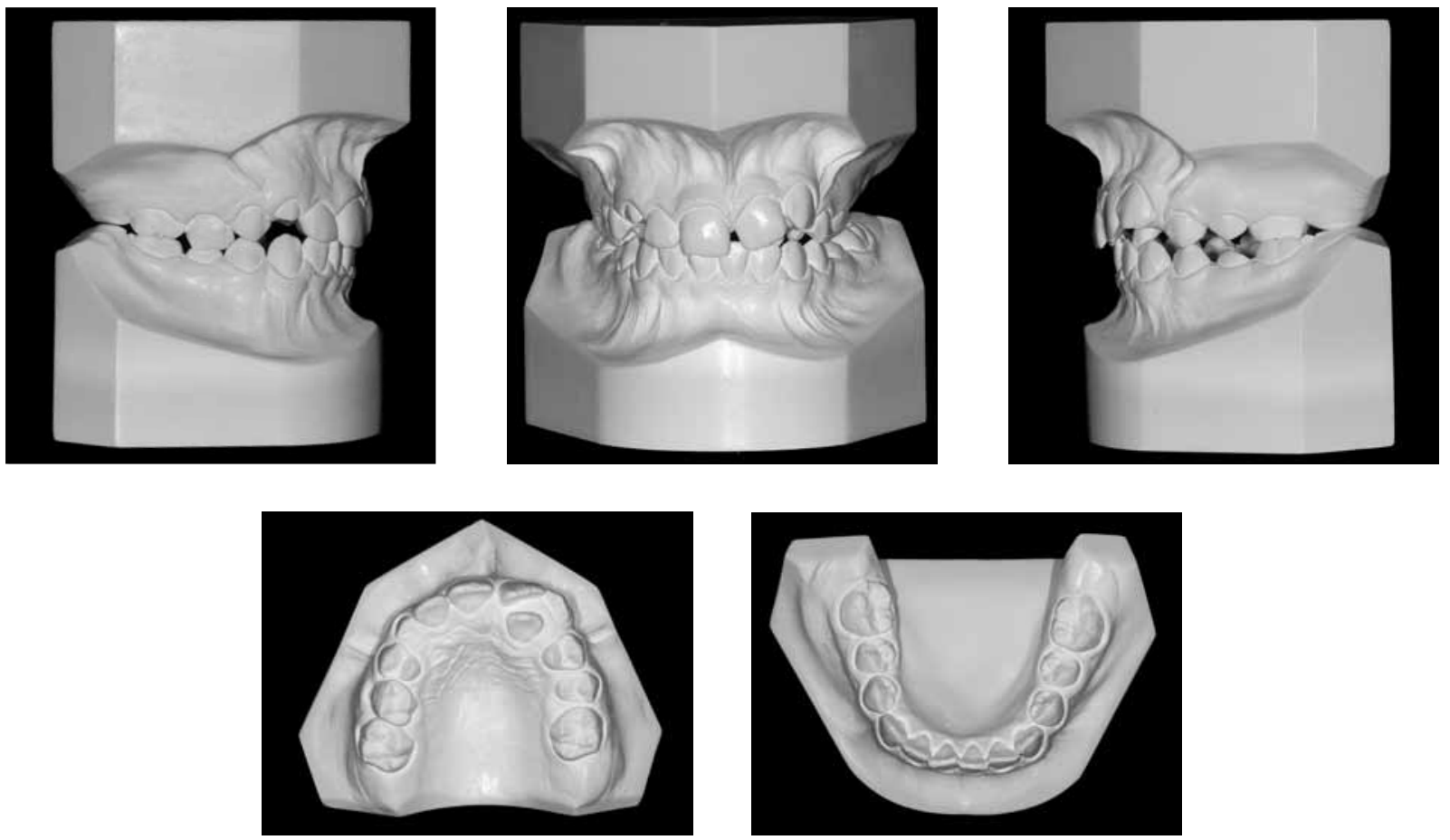

FIGURE 2 - Initial dental casts. 

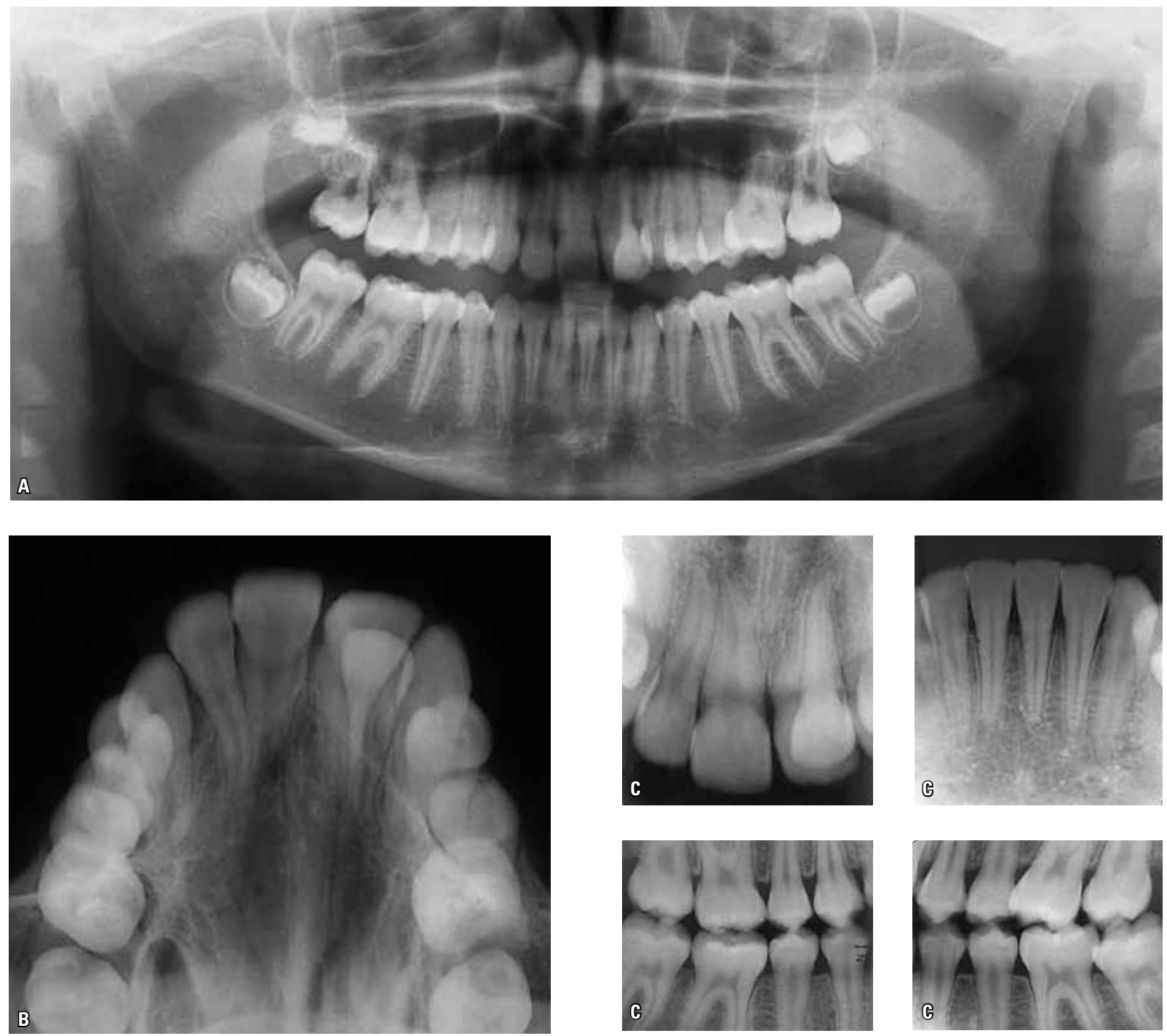

FIGURE 3 - Panoramic (A), upper occlusal (B), anterior region periapicals and posterior region interproximal (C) radiographs.
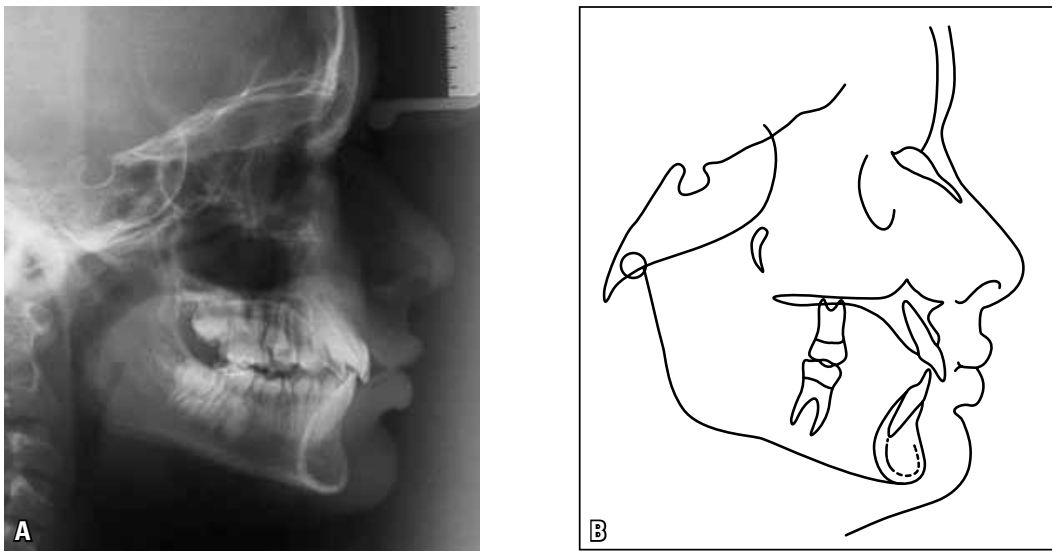

FIGURE 4 - Initial profile cephalometric radiograph (A) and cephalometric tracing (B). 
disocclusion in mandibular movements. ${ }^{3}$ The best posterior intercuspation, with simultaneous and equivalent bilateral contacts should also be present, despite the asymmetric molar relation, with occlusal contacts on the right side and distoclusion on the left side.

\section{TREATMENT PLAN}

The treatment plan involved palatal expansion, with an overcorrection of the posterior crossbite-contacts edge to edge of the upper palatal cusps and lower buccal cusps. ${ }^{4}$ Subsequently, an upper removable appliance with an anterior plateau, simultaneously to a lower fixed appliance, including the second molars, would be used in order to accomplish more effective dental leveling and alignment. Upper fixed appliance would be finally included and the request for extraction of the upper left second premolar would be made.

After upper arch leveling, the upper left canine would be distalized using a compression coil spring. The eventual need for an upper frenectomy was also included in the treatment plan. After the $0.019 \times 0.025$-in stainless steel upper and lower archwires, the upper left lateral incisor had its orthodontic traction planned with an overlapped TMA $0.017 \times 0.025$-in wire section. By then, Class II intermaxillary elastics would be attached to the right side.

Manipulation in centric relation at every appointment would be an important reference and to avoid dual bite. ${ }^{1}$ Posterior intercuspation and anterior guidance would be finally established with $0.019 \times 0.025$-in delta loop stainless steel wires in both arches, closing remaining spaces. At the finishing stage priority would be given to control the torque of the upper left lateral incisor. Upper wraparound and lower lingual arch retentions were planned, bonded to the canines by a 0.028 -in stainless steel wire.

\section{TREATMENT EVOLUTION}

A modified Haas appliance was installed for palatal expansion, fixed to teeth \#16 and \#26 by wires welded to bands, and to teeth \#14 and \#24 with wires bonded to the buccal surface by composite resin and with a $7-\mathrm{mm}$ screw, activated twice a day and stabilized after the planed overcorrection was reached (Fig 5).

After four months, the Haas appliance was replaced by a removable appliance with an anterior plateau, creating a posterior disocclusion to accelerate the effects of the fixed appliance on
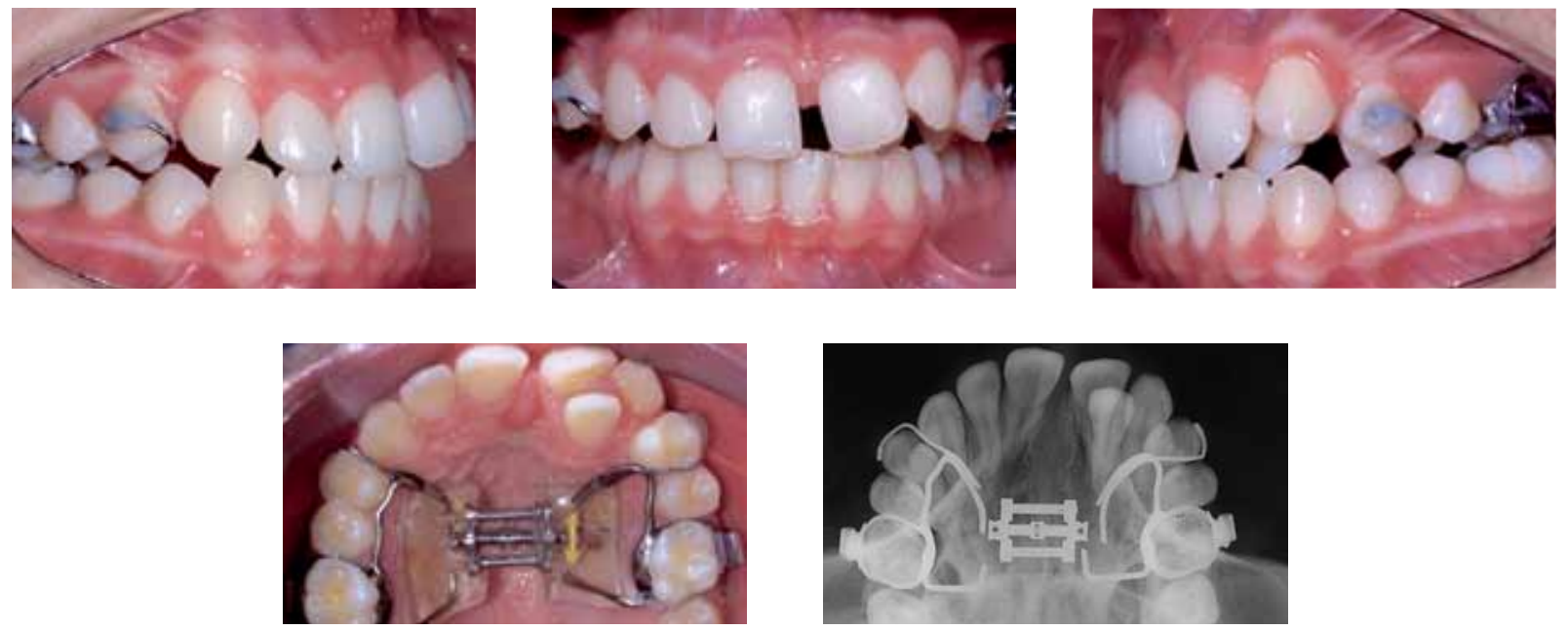

FIGURE 5 - Photographs and occlusal radiograph after palatal expansion. 
the lower arch for correction of the severe overbite. In this case, the lower second molars were included in the appliance, with leveling from 0.014-in through 0.020-in archwires.

Extraction of the upper left second premolar was requested in the upper arch. The decision for the extraction of a single premolar was directly linked to the facial profile of the patient. The intention was to avoid retraction of anterior teeth, keeping the lips from retruding. It should be taken into account that the nose and chin would have become more prominent in the face due to the normal ageing pattern of brachyfacial patients. ${ }^{9}$ The fibrous upper lip frenum was also removed.

From 0.018-in upper archwire onwards, chain elastics were used for the distal movement of the upper left premolar and a NiTi open coil spring was incorporated between upper left central incisor and canine, in order to correct both midline deviation and canine distalization (Fig 6). For the alignment of the upper left lateral incisor, a titanium-molybdenum alloy (TMA) 0.017 x 0.025-in archwire segment was used with a cantilever rectangular loop as a means to achieve root control since the beginning of the movement.

A $0.019 \times 0.025$-in archwire was initially attached to the lower arch in order to function as anchorage for the Class II intermaxillary elastics on the right side. Subsequently, a rectangular $0.019 \times 0.025$-in delta loop archwire was attached to the upper arch to close remaining spaces and for root control during finishing stage. The wraparound retention appliance was used on the upper arch and a fixed retainer bonded to canines on the lower arch.

For a better intercuspation on the left side, where there was a molar distoclusion relation, a mesial rotation on the upper left first molar was established. ${ }^{1,2}$

\section{TREATMENT RESULTS}

Considering the patient's final records, an improvement of the transverse dimension of the maxillary arch was noticed, which was crucial for the left side crossbite correction.
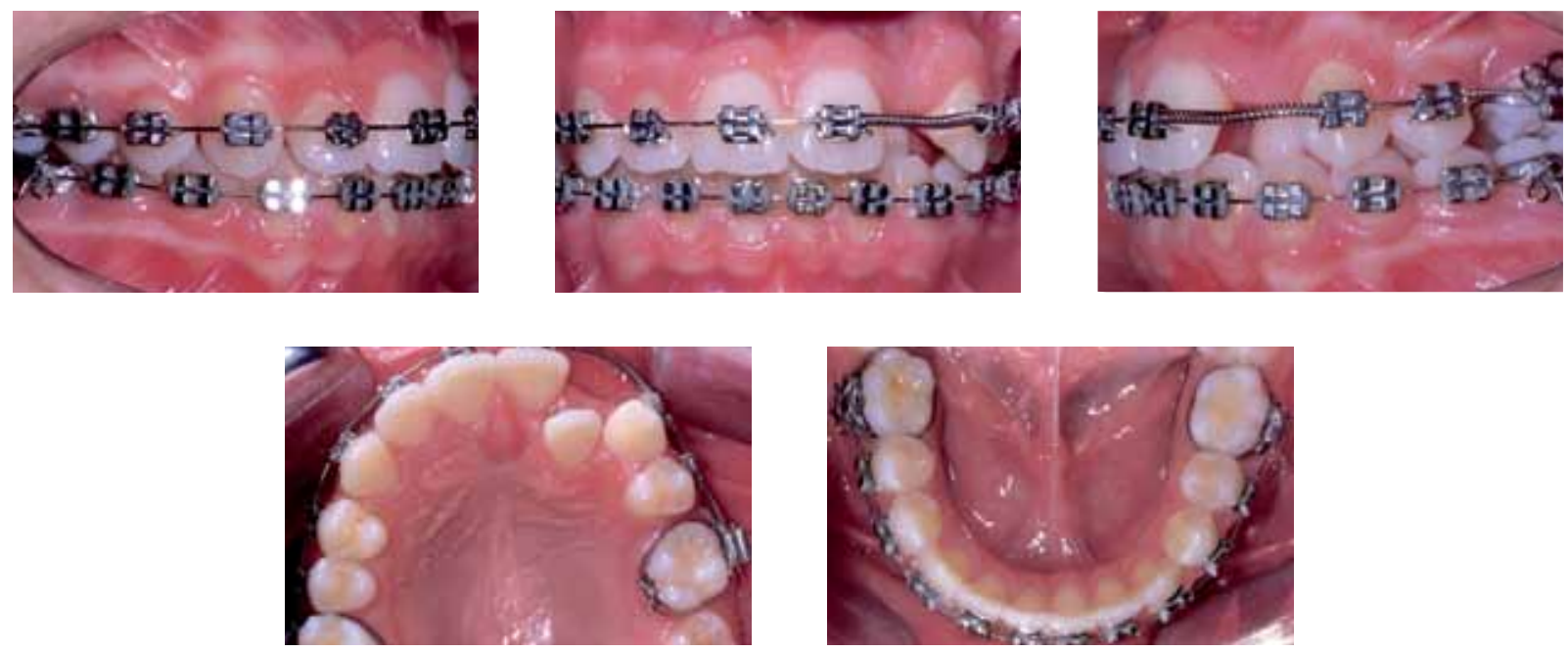

FIGURE 6 - Photographs of the space opening with the compressing coil spring, making upper left lateral incisor movement possible. 
The skeletal effect of expansion obtained with the Haas appliance is widely demonstrated throughout the literature. ${ }^{4,5}$ The stability of results is easily explained by the position of teeth over the bony base and by the placement of muscle attachments away from teeth crowns. ${ }^{5}$ On top of that, there is the aesthetic effect of the increased width of the dental arch, reaching up to $6 \mathrm{~mm}$ in this case, decreasing the buccal corridor and creating a wider and more pleasant smile. ${ }^{9}$ Intercanine width was maintained on the lower arch with a slight increase in the intermolar width, resulting from lower right first molar torque adjustment (Fig 7).
Left intercuspation had to be adapted to the upper second premolar extraction option on this side. In this case, the molar relation was in distoclusion, which required special adjustments: 1- The upper first molar was finished with a mesial rotation so that the mesiobuccal cusp would touch the distal slope of the buccal cusp of the lower left second premolar, given the smaller dimension of the latter (Fig 8); 2- In order to compensate the larger mesiodistal width of the molar, some stripping was needed on both canine and first molar; and 3- Enameloplasty on the occlusal slope of the mesiobuccal cusp of the first molar, as prescribed by Brandão and Brandão. ${ }^{1}$
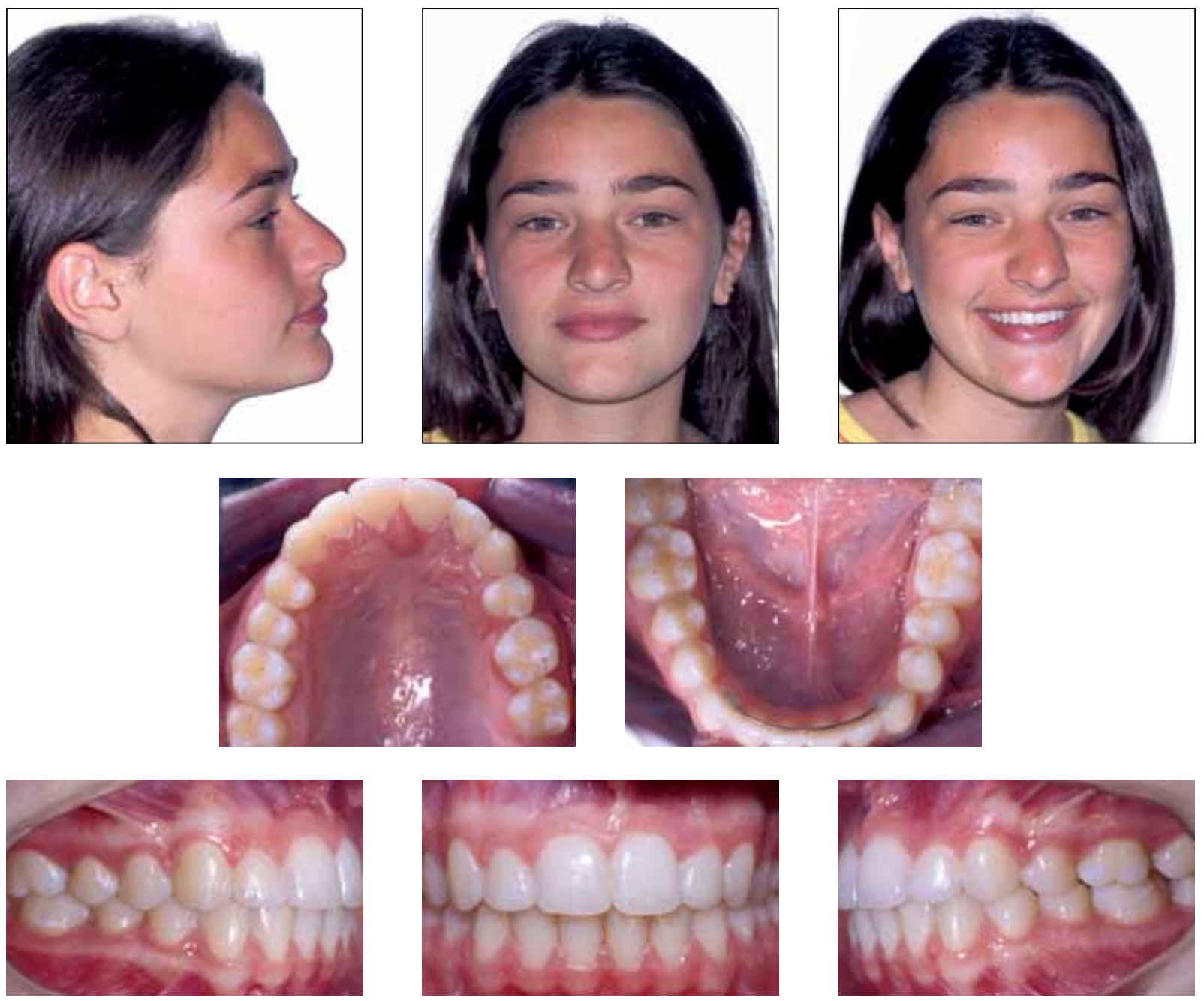

FIGURE 7 - Final facial and intraoral photographs. 
On the right side, a normal occlusion relation was established between molars, as well as the proper relation of the mesiodistal inclined planes of the cusps of other teeth. On both sides, normal occlusion was obtained between canines, which led to ideal overjet and overbite, plus the immediate disocclusion during mandible excursion movements. ${ }^{1,3}$

Radiographic records revealed parallel roots and integrity of almost all teeth and alveolar bone ridges. The slight round shape of root apexes is compatible to the orthodontic treatment. A larger root resorption was observed on the mesial root of tooth \#26, due to the proximity with the maxillary sinus cortical plates associated to dental movement (Fig 9).

Cephalometric measurements and superimpositions demonstrate a significant mandibular growth, as expected according to the facial profile presented by the patient. The decision for extracting a single second premolar was shown to be efficient in avoiding the retraction of anterior teeth and worsening of the facial profile (Fig 11 and Table 1). ${ }^{9}$

But the better effect of the biomechanics applied was seen in the smile. Midline correction, associated to the improvement on upper anterior teeth has set harmony to the smile (Fig 12). The symmetry obtained when exhibiting anterior teeth as well as the dominance of central incisors were also noticeable. ${ }^{8}$ On the other hand, there was an increase in the concavity of the facial profile due to expressive mandibular and nasal growth, greater than the one presented by the nasomaxillary complex (Fig 10). Upper incisor projection to compensate this lack of proportion between maxillary and mandibular structures did not alter this tendency. A good gingival contour was obtained and, together with the smile curve that delineates the lower lip curvature, they define a pleasant smile presenting dominance of upper central incisors. ${ }^{6,7}$
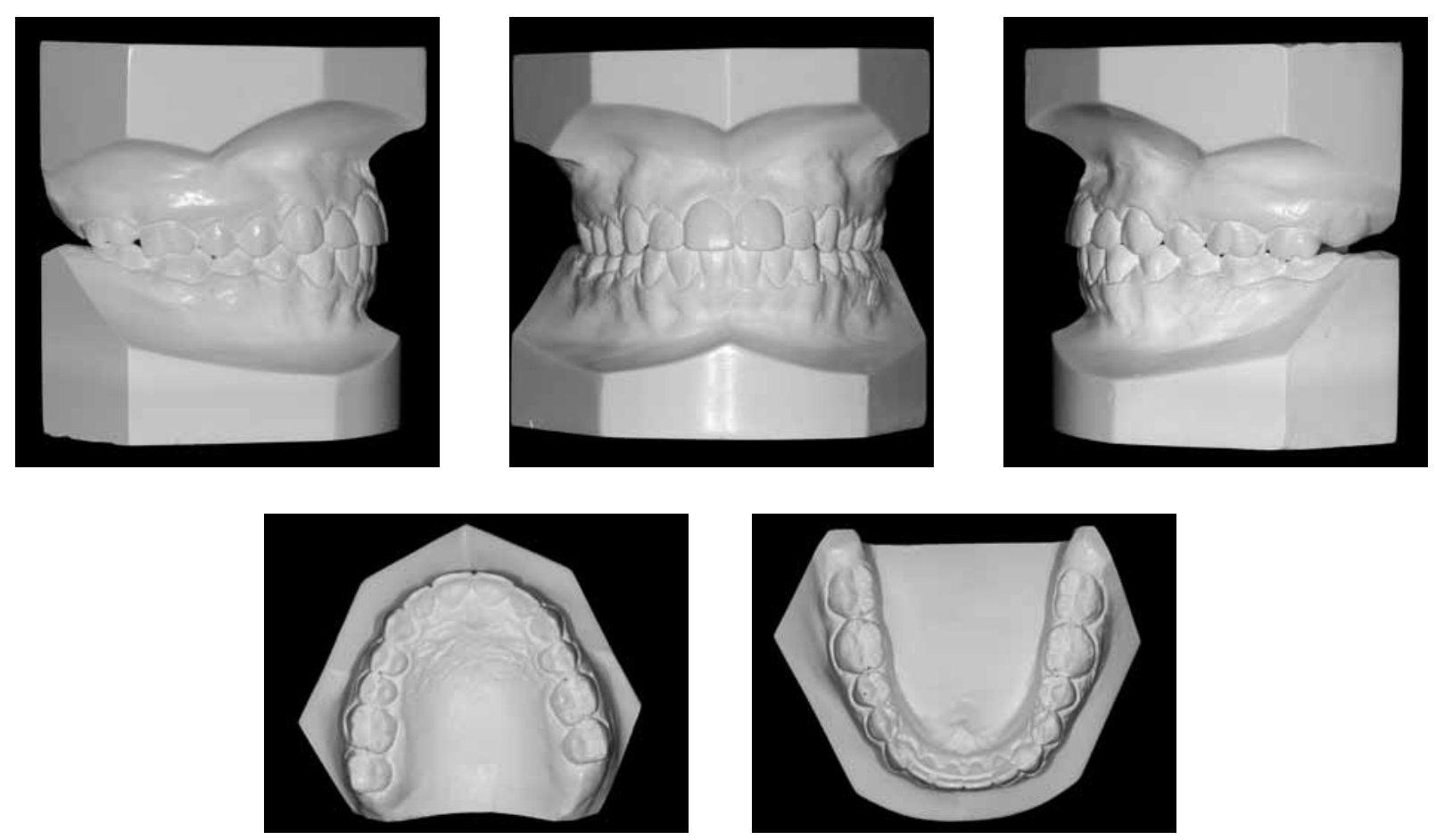

FIGURE 8 - Final dental casts. 

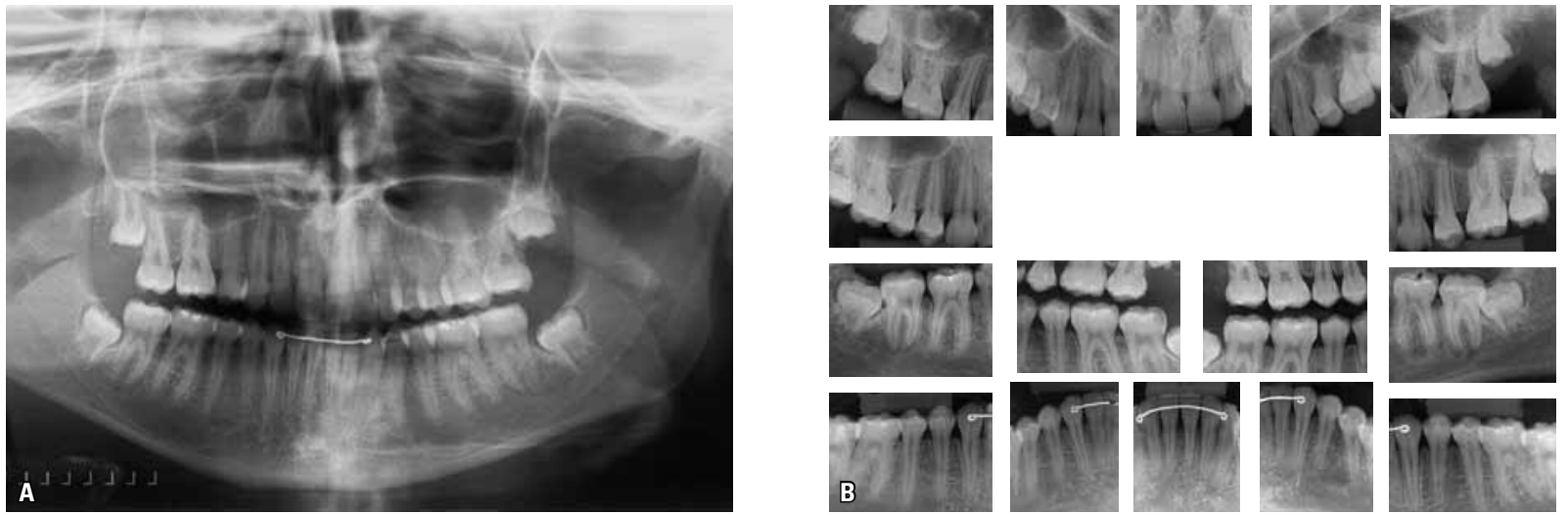

FIGURE 9 - Final radiographs: panoramic (A) and periapical (B).
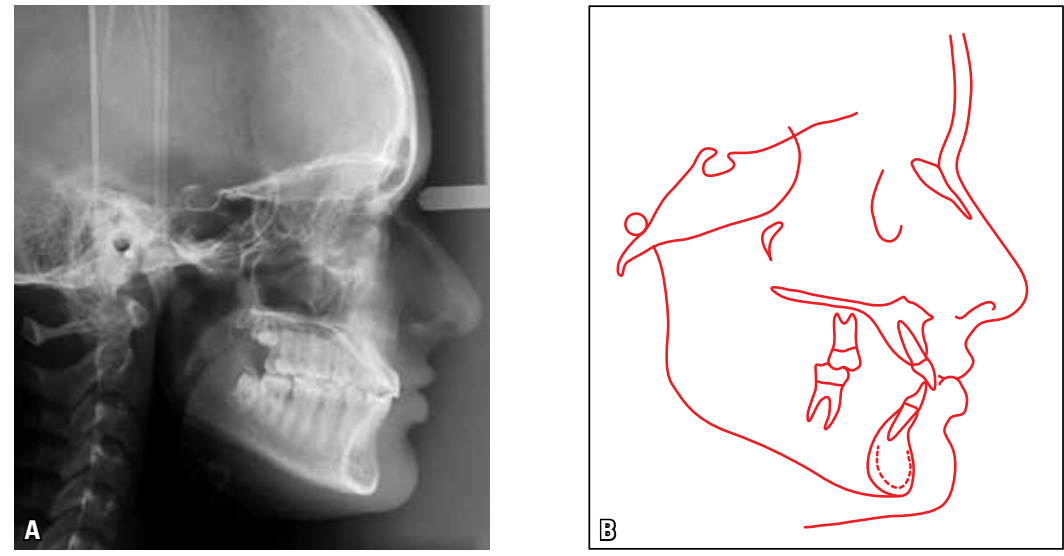

FIGURE 10 - Final lateral cephalometric radiograph (A) and tracing (B).
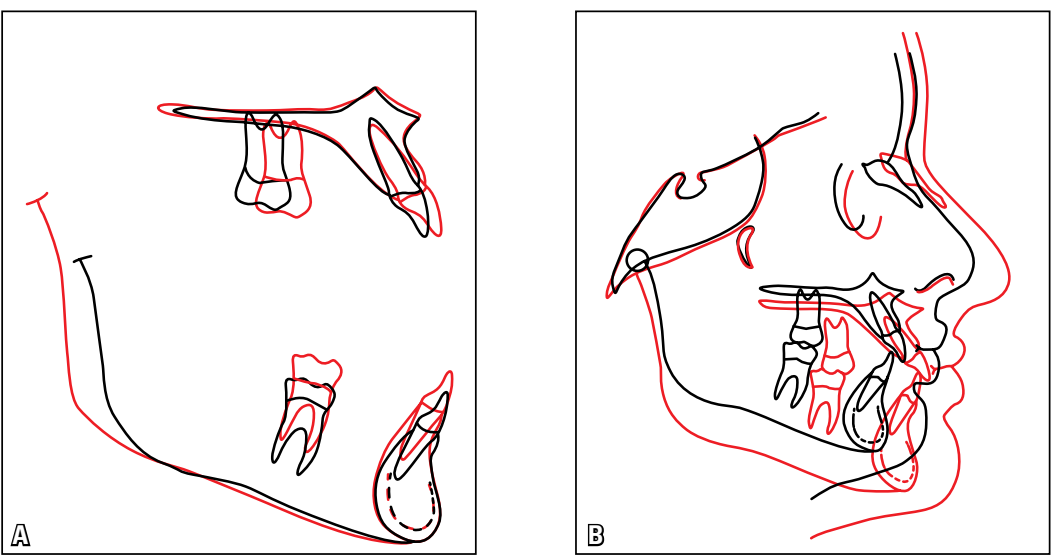

FIGURE 11 - Total superimposition (A) and partial superimposition (B) of both initial (black) and final (red) cephalometric tracings. 
TABLE 1 - Summary of cephalometric measurements.

\begin{tabular}{|c|c|c|c|c|c|}
\hline & MEASURES & Normal & $\mathbf{A}$ & B & $\begin{array}{c}\text { A/B } \\
\text { DIFFERENCE }\end{array}$ \\
\hline \multirow{8}{*}{ 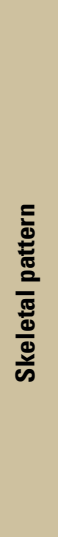 } & SNA (Steiner) & $82^{\circ}$ & $83^{\circ}$ & $83^{\circ}$ & 0 \\
\hline & SNB (Steiner) & $80^{\circ}$ & $81^{\circ}$ & $83^{\circ}$ & 2 \\
\hline & ANB (Steiner) & $2^{\circ}$ & $2^{\circ}$ & $0^{\circ}$ & 2 \\
\hline & Convexity angle (Downs) & $0^{\circ}$ & $3^{\circ}$ & $-2^{\circ}$ & 5 \\
\hline & Y axis angle (Downs) & $59^{\circ}$ & $58^{\circ}$ & $56^{\circ}$ & 2 \\
\hline & Facial angle (Downs) & $87^{\circ}$ & $86^{\circ}$ & $91^{\circ}$ & 5 \\
\hline & SN-GoGn (Steiner) & $32^{\circ}$ & $24^{\circ}$ & $23^{\circ}$ & 1 \\
\hline & FMA (Tweed) & $25^{\circ}$ & $18^{\circ}$ & $17^{\circ}$ & 1 \\
\hline \multirow{7}{*}{ 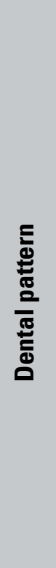 } & IMPA (Tweed) & $90^{\circ}$ & $105^{\circ}$ & $100^{\circ}$ & 5 \\
\hline & $\underline{1}$ - NA (degrees) (Steiner) & $22^{\circ}$ & $24^{\circ}$ & $32^{\circ}$ & 8 \\
\hline & $\underline{1}-\mathrm{NA}(\mathrm{mm})($ Steiner $)$ & $4 \mathrm{~mm}$ & $5 \mathrm{~mm}$ & $6 \mathrm{~mm}$ & 1 \\
\hline & $\overline{1}-$ NB (degrees) (Steiner) & $25^{\circ}$ & $30^{\circ}$ & $25^{\circ}$ & 5 \\
\hline & $\overline{1}-\mathrm{NB}(\mathrm{mm})$ (Steiner) & $4 \mathrm{~mm}$ & $4 \mathrm{~mm}$ & $4 \mathrm{~mm}$ & 0 \\
\hline & $\frac{1}{1}-$ Interincisal angle (Downs) & $130^{\circ}$ & $125^{\circ}$ & $121^{\circ}$ & 4 \\
\hline & $\overline{1}-\mathrm{APo}(\mathrm{mm})$ (Ricketts) & $1 \mathrm{~mm}$ & $2 \mathrm{~mm}$ & $3 \mathrm{~mm}$ & 1 \\
\hline \multirow{2}{*}{$\frac{\text { 는 }}{\text { 는 }}$} & Upper lip - S line (Steiner) & $0 \mathrm{~mm}$ & $0 \mathrm{~mm}$ & $-4 \mathrm{~mm}$ & 4 \\
\hline & Lower lip - S line (Steiner) & $0 \mathrm{~mm}$ & $0 \mathrm{~mm}$ & $-3 \mathrm{~mm}$ & 3 \\
\hline
\end{tabular}
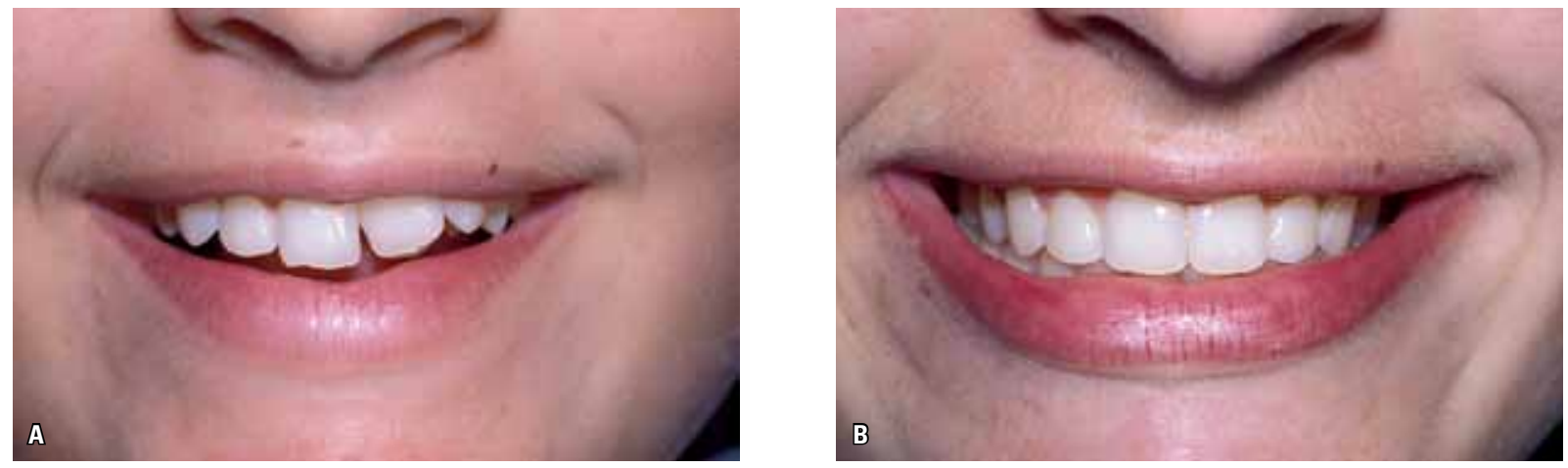

FIGURE 12 - Initial (A) and final (B) smile photographs with the aesthetic results obtained, showing a broader smile and a smaller buccal corridor. 


\section{FINAL REMARKS}

In this case, skeletal approach and growth use occurred in the transverse aspect. There was significant space gain and simplification of the problem since the maxillary constriction was corrected. What escaped the skeletal action did happen as a consequence of the dental movement, especially after molar dentoalveolar corrections and compensations. The projection of upper incisors was needed in order to offset a smaller anteroposterior growth of the maxilla in relation to the mandible. On the other hand, the strategy of fewer extractions, maintaining the posterior asymmetry and upper incisor proclination seem to have been the best option when compared to a greater number of extractions or distalizing appliances that could have caused even further lip retraction, compromising facial aesthetics. ${ }^{9}$ The stability expected for this treatment is directly linked to the palatal expansion, of utmost importance for the successful management of this case. ${ }^{5}$

\section{REFERENCES}

1. Brandão RCB, Brandão LBC. Ajuste oclusal na Ortodontia: por que, quando e como? Rev Dental Press Ortod Ortop Facial. 2008;13(3):124-156.

2. Brandão RCB. Entrevista. Rev Dental Press Ortod Ortop Facial. 2009;14(6):19-41.

3. Dawson PE. Oclusão funcional: da ATM ao desenho do sorriso. St. Louis: C.V. Mosby; 2008.

4. Haas AJ. Palatal expansion: just the beginning of dentofacial orthopedics. Am J Orthod. 1970;57(3):219-55.

5. Haas AJ. Long-term post-treatment evaluation of rapid palatal expansion. Angle Orthod. 1980;50(3):189-217.

6. Kokich VG. Esthetics: the orthodontic-periodontic restorative connection. Semin Orthod. 1996;2(1):21-30.

7. Kokich VG. Excellence in finishing: modifications for the perio-restorative patient. Semin Orthod. 2003;9(3):184-203.
8. Sarver DM. The importance of incisor positioning in the esthetic smile arc. Am J Orthod Dentofacial Orthop. 2001; 120(2):98-111.

9. Sarver DM. Soft-tissue-based diagnosis and treatment planning. Clinical impressions. 2005;14(1):21-6.

Submitted: October 25, 2011

Revised and accepted: November 13, 2011

Contact address

Roberto Carlos Bodart Brandão

Av. Américo Buaiz, 501/1007 - Enseada da Praia do Suá

Zip code: 29.050-911 - Vitória/ES, Brazil

E-mail: roberto@ortobrandao.com.br 